\title{
The Crisis in Psychopharmacology Provides an Opportunity for Neuroregulation Treatments to Gain Widespread Acceptance
}

\author{
H. Edmund Pigott \\ Private practice
}

\begin{abstract}
Psychopharmacology is in crisis due to the increasing recognition that it does not work as claimed and has failed to meaningfully improve outcomes over what they were in the 1950 s and '60s. Though still widely promoted to the public, the chemical imbalance theory of major mental health disorders is now openly acknowledged as not accurate by leading psychiatrists, thereby undermining the rational for this approach to care. A series of large comparative effectiveness studies funded by the National Institute of Mental Health (NIMH) were each essentially failed trials with disappointing results and found that second-generation psychotropic medications were no more effective than their first-generation cousins. The evidence from several of these studies are reviewed within the scope of major depression and attention-deficit/hyperactivity disorders, and then compared to research on promising neuroregulation treatments. The author then makes recommendations for neuroregulation clinicians to avoid a crisis similar to that experienced in psychopharmacology today.
\end{abstract}

Keywords: psychopharmacology; neurofeedback; depression; STAR*D; MTA cooperative study; ADHD

Citation: Pigott, H. E. (2017). The crisis in psychopharmacology provides an opportunity for neuroregulation treatments to gain widespread acceptance. NeuroRegulation, 4(1), 28-36. http://dx.doi.org/10.15540/nr.4.1.28

*Address correspondence to: $\mathrm{H}$. Edmund Pigott, PhD, BCN, $430 \mathrm{~N}$. Edited by:

Lyra Circle, Juno Beach, FL 33408, USA. Email: Rex L. Cannon, PhD, Neurogifted, Boynton Beach, Florida, USA

pathware@erols.com

Copyright: @ 2017. Pigott. This is an Open Access article distributed under the terms of the Creative Commons Attribution License (CCBY).

\section{Reviewed by:}

Randall Lyle, PhD, Mount Mercy University, Cedar Rapids, lowa, USA

\section{Background}

In the attempt to provide evidence-based guidance to clinical practice and improve outcomes, the National Institute of Mental Health (NIMH) funded a series of large comparative effectiveness studies for attention-deficit/hyperactivity disorder (ADHD), the multimodal treatment of attention deficit hyperactivity disorder (MTA cooperative study): cost \$21 million (Jensen et al., 2007; Molina et al., 2009; MTA Cooperative Group, 2004a, 2004b); bipolar disorder, the systematic treatment enhancement program for bipolar disorder (STEP-BD): \$26 million (Bowden et al., 2012); major depression, sequenced treatment alternatives to relieve depression (STAR*D): \$35 million (Fava et al., 2003; Rush, 2002; Rush et al., 2006; Trivedi et al., 2006; Trivedi, Stegman, Rush, Wisniewski, \& Nierenberg, 2002); and schizophrenia, clinical antipsychotic trials of intervention effectiveness (CATIE): $\$ 72$ million
(Lieberman et al., 2005), among other well-funded efforts. In an editorial, DePaulo (2006), past chairman of psychiatry at Johns Hopkins, termed these studies as effectiveness-plus because each used the best available treatment methods to optimize outcomes. Furthermore, there was no blinding of treatments in each of these trials, thus taking advantage of nonspecific placebo effects, which inflate outcomes. Despite the costs, and investigators' best efforts, each of these studies were essentially failed trials with outcomes far less than expected. DePaulo noted how the studies taken together "underline the suggestion that modern pharmacological treatments may be no more beneficial than older ones, despite their added cost" (2006, p. 175). Similarly, former NIMH Director Insel (2009) observed that in each of these effectiveness-plus studies second-generation psychotropic medications were no better than their first-generation cousins and then went on to 
acknowledge, "The unfortunate reality is that current medications help too few people to get better and very few people to get well" (p. 704).

As evidenced in a 2012 editorial by Fibiger, former Vice President of Neuroscience at Eli Lilly, DePaulo (2006) and Insel (2009) are not alone in acknowledging the current state of psychopharmacology. Fibiger writes the following:

Psychopharmacology is in crisis. The data are in, and it is clear that a massive experiment has failed: despite decades of research and billions of dollars invested, not a single mechanistically novel drug has reached the psychiatric market in more than 30 years. Indeed, despite enormous effort, the field has not been able to escape the "me too/me (questionably) better" straightjacket (p. 649).

Fibiger (2012) goes on to note that each of psychiatry's classes of medication were discovered by "serendipitous clinical observation" (p. 649) and would likely not have been discovered using current drug discovery strategies. Thus, concluding that:

What the field has been doing for the past 3 or 4 decades has failed to generate effective, mechanistically novel psychopharmaceuticals...there is no choice but to make changes in how we approach the study of disease mechanisms, drug discovery, and development in psychiatry. This will require major investments in neuroscience research, humility in the face of our ignorance, and a willingness to consider fundamental reconceptualizations of psychiatry itself (p. 650).

Hyman (2012), another former NIMH Director, acknowledges how the initial serendipitous findings from the 1950s "motivated path-breaking research on neurotransmitter release, receptors, and transporters" but "what has happened-or rather not happened-in the intervening half-century was as unexpected as the initial spate of discoveries" (p. 1). Hyman goes on to observe that:

The molecular targets of all of today's approved psychiatric drugs are the same as the targets of their pre-1960 prototypes and their mechanisms of action are not understood beyond a few initial molecular events...By capturing the imagination of researchers to excess, however, and in the absence of other robust biological tools to probe brain function, these drugs may have proved something of a scientific curse (p. 12).

The widely acknowledged failure to improve psychopharmacology outcomes has gotten so bad that not only are academic psychiatrists actively disavowing the neurochemical imbalance theory of major mental health disorders, but some apologists claim that it was never even a theory held by responsible psychiatrists in the first place. Pies (2011), Editor in Chief Emeritus of Psychiatric Times, writes, "In truth, the 'chemical imbalance' notion was always a kind of urban legend-never a theory seriously propounded by well-informed psychiatrists" (p. 1).

Pies concludes his editorial by stating that "the legend of the 'chemical imbalance' should be consigned to the dust-bin of ill-informed and malicious caricatures," as though this horse that biological psychiatry rode to prominence-backed by billions in taxpayer- and industry-funded research and many billions more in pharmaceutical companies' deceptive marketing efforts-was not only a half-century long fool's errand/scientific curse "chasing down chemical imbalances that don't exist" (Greenberg, 2013, p. 6) but it is now a "malicious caricature" (Pies, 2011, p. 2) to expose this fact. In response to Pies (2011), Hickey (2014) provides extensive documentation going back to the early 1970s of eminent biological psychiatrists as well as the American Psychiatric Association itself propagating the chemical imbalance theory of mental illness and how this theory is featured prominently on numerous authoritative websites as well as on TV today.

\section{Major Depression}

The STAR*D study (Fava et al., 2003; Rush, 2002; Rush et al., 2006; Trivedi et al., 2006) is the largest antidepressant study ever conducted with over 120 journal articles published by study investigators. STAR*D enrolled 4,041 patients diagnosed with major depression, including patients with comorbid conditions; thereby increasing the generalizability of its findings, while also providing 12 months of free follow-up care to monitor the durability of treatment effects. The study provided up to four drug trials per patient with the hope of being able to give guidance in selecting the best next-step treatment for the many patients who fail to get sufficient relief from their initial antidepressant and subsequent trials. Each drug trial/step lasted up to 12 weeks with an 
additional 2 weeks added on for those patients deemed close to remission. Antidepressants were administered using measurement-based care that involved assessing symptoms and side effects at each visit to guide aggressive medication dosing in order to ensure that the likelihood of achieving remission was maximized and that those who did not reach remission were truly resistant to the medication (Trivedi et al., 2006).

The researchers allowed patients to select treatment options for randomization in steps 2-4 "to empower patients, strengthen the therapeutic alliance, optimize treatment adherence, and improve outcome" (Fava et al., 2003, p. 483) and evaluated the relative effectiveness of 11 pharmacologically distinct drug/drug combination treatments in five head-to-head comparisons. Patients who achieved remission during any step were encouraged to enter the 12 months of free follow-up care. The follow-up protocol "strongly recommended that participants continue the previously effective acute treatment medication(s) at the doses used in acute treatment" but treating physicians were allowed to make "any psychotherapy, medication, or medication dose change" they deemed necessary to sustain remission during follow-up, including scheduling additional visits if depressive symptoms returned and/or intolerable side effects emerged (Rush et al., 2006, p.1908).

In different publications, the author among others have criticized the $S T A R^{*} D$ investigators for extensive evidence of researcher bias that significantly inflated their reports of outcomes (e.g., Pigott, 2011; Pigott, 2015; Pigott, Leventhal, Alter, \& Boren, 2010). Despite it being over six years since the Pigott et al. (2010) publication, STAR*D's researchers have still not offered a defense for their biases documented therein. In 2011, Pigott and Alter published a response to two letters commenting on the first STAR*D article. Neither letter questioned the Pigott et al. analysis, though STAR*D's researchers could easily have published a counterargument since one of its principal investigators was an associate editor for the journal. This did not happen; instead the researchers continued publishing articles untethered to their prespecified analytic plan and primary measure.

Figure 1 is a comparison between $\operatorname{STAR}^{*} D$ researchers' predicated success rate, their post hoc concocted theoretical success rate, and STAR*D's actual step-by-step success rates (Pigott, 2015). The predicated success rate is taken from Figure 7 in STAR*D's Research Protocol's step-by-step predictions of dropout and the number of patients who would have a satisfactory response and enter follow-up (Rush, 2002). The author obtained the protocol through a Freedom of Information Act request. The predictions were made by STAR*D's three most highly published researchers who had over 1,900 published studies between them. Regarding the predictions, the protocol states that they were arrived at based on the independent estimates of Drs. Fava, Rush, and Thase, informed by the results of published randomized controlled trials (Rush, 2002).

While these predictions' purpose was to estimate the number of continuing patients available for randomization in steps $2-4$ and to ensure adequate statistical power for the planned comparisons, at the metalevel, these predictions are the central hypothesis STAR*D tested by assessing how well these experts could predict the aggregate step-bystep outcomes from optimally delivered antidepressant drug treatment. Evaluating these predictions is important in learning the correct lessons from $S T A R^{*} D$, since there were no significant group differences between any of the 11 drug/drug combination treatments, even though there was adequate statistical power to discern differences, if any existed. Furthermore, no post hoc secondary analyses have yielded significant predictors of outcomes between the pharmacologically distinct treatments. Therefore, this $\$ 35$ million taxpayer-funded study provides no next-step guidance to give hope for improving outcomes from the optimal administration of antidepressants beyond that found in the study itself.

In STAR*D's summary article the researchers calculated a "theoretical cumulative remission rate of $67 \%$ " with the scientifically baseless provisos that "this estimate assumes no dropouts, and it assumes that those who exited the study would have had the same remission rates as those who stayed in the protocol" (Rush et al., 2006, p. 1910-1911). As Pigott et al. (2010) document, however, the researchers' assumptions in calculating their theoretical remission rate are simply not true in the real world-and was certainly not true in $S T A R^{*} D$, since more patients dropped out in each step than remitted. Today, STAR ${ }^{\star} D$ researchers' baseless provisos are commonly dropped when portraying its findings. For example, an American Journal of Psychiatry editorial states STAR*D found, "after four optimized, well-delivered treatments, approximately $70 \%$ of patients achieve remission" (Greden, 2013, p. 580), as though this is a factual statement of what occurred. 


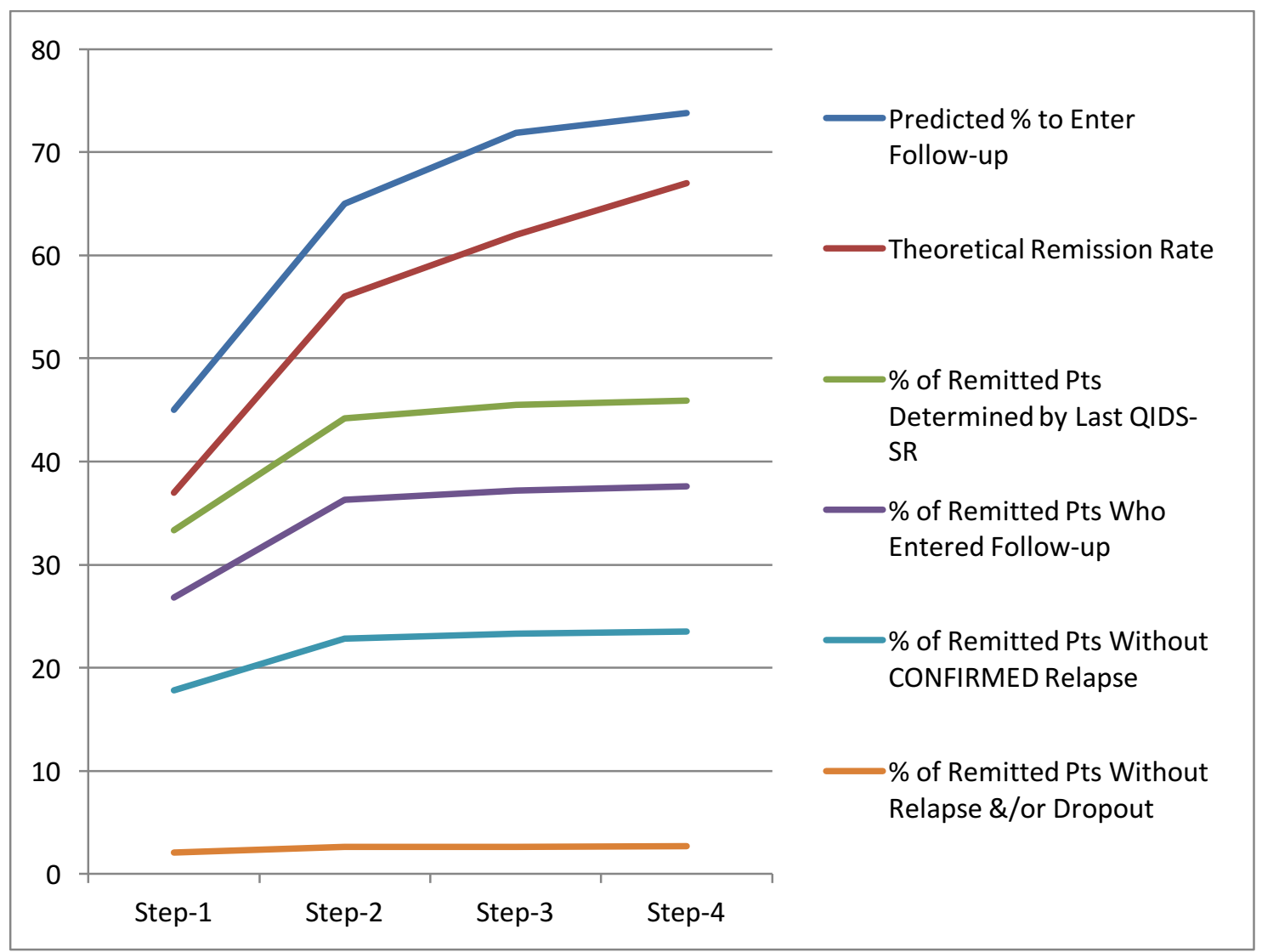

Figure 1. Comparison between predicted, theoretical, and actual step-by-step success rates (Pigott, 2015, used with permission).

As is evident in Figure 1, STAR*D's predicted $(73.8 \%)$ and theoretical $(67.0 \%)$ success rates are similar, yet highly divergent from what actually occurred after up to four drug trials, in that the cumulative percent of patients who had a remission was only $45.9 \%$ and, by step 4 , the cumulative percent of patients who had a remission and entered free follow-up care was a mere $37.6 \%$.

The data $S_{T A R}{ }^{D} \mathrm{investigators}$ provide for accessing the durability of treatment gains are even more discouraging. For step 1 , only $17.8 \%$ of patients had a remission and during follow-up did not have a confirmed relapse. After up to four rounds of antidepressant drug/drug combination treatments, the cumulative rate of patients who did not have a confirmed relapse improved to only 23.5\% (and this from optimal acute and follow-up care). When dropout is added, the durability of treatment effects is even paltrier; only $2.7 \%$ of the 4,041 enrolled patients had a remission after up to four rounds of optimal care and neither relapsed nor dropped out during the 12 months of free follow-up services.
On the other hand, whereas psychopharmacology has not worked as claimed, neurofeedback (NFB) and other neuroregulation strategies are suitable alternative treatments (Choi et al., 2011; Peeters et al., 2014). While there are many studies demonstrating the effectiveness of these strategies for treating major depression, the Cantor and Stevens (2009) study is exemplary in its experimental design, use of quantitative electroencephalography (qEEG) biomarkers of depression for study inclusion, and incorporation of both neurophysiologic and rating scale measures to evaluate outcomes. The researchers randomly assigned 16 treatment-resistant depressed patients into two groups of eight (simulated and active $14 \mathrm{~Hz}$ audio-visual entrainment) in a crossover research design. Patients received either simulated or active auditory-visual EEG entrainment (AVE) 5 days per week for 4 weeks and then crossed over. As reported by the researchers, "all participants were required to have increased frontal relative alpha or increased relative frontal beta on a neurometric qEEG evaluation to qualify for the study based on 
previous studies indicating such deviations in depression samples" (p. 102). Key findings were:

- Significant improvements on the Beck Depression Inventory-II (BDI) and neurophysiologic measures were only associated with active AVE treatment $(p>$ $.01)$

- AVE resulted in $50 \%$ or greater improvement on the BDI for all patients;

- $\quad$ AVE resulted in significant EEG changes in cortical regions associated with mood regulation; and

- AVE treatment gains were sustained for 1 month following termination for the group who received AVE first.

The opportunity for neuroregulation strategies to have a significant impact on the treatment of major depression is not going away, only increasing. A recent article by Kelland (2017) reports that experts believe it will be more than decade "before any new generation of antidepressants comes to market" and cites the Kantor et al. (2015) study finding a neardoubling in the number of American adults taking antidepressants between 1999 and 2012, rising to 13.0 percent from 6.9 .

Echoing the observations of Fibiger (2012) and Hyman (2012), Kelland's article quotes Oxford psychiatry professor Guy Goodwin acknowledging that psychopharmacology's lack of progress is "partly a failure of science, to be frank...Scientists have to get more of an understanding about how these things actually work before we can then propose ways to improve them." Despite negligible research funding, the neuroregulation field is in many ways ahead of psychopharmacology in finding effective treatments in that we have validated $q E E G$ biomarkers for depression (e.g., John, Prichep, Friedman \& Eastman, 1988; John et al., 2007) and both NFB and neurostimulation-based strategies to correct them; to date, there is nothing comparable in psychopharmacology.

\section{ADHD}

There have been two large NIMH-funded ADHD studies that included long-term follow-up assessments, the MTA Cooperative study (Jensen at al., 2007; Molina et al., 2009; MTA Cooperative Group, 1999, 2004a, 2004b) and the Preschool Attention-Deficit/Hyperactivity Disorder Treatment Study (PATS; Riddle et al., 2013). Pigott and Cannon (2014) provide a detailed critique and deconstruction of these studies. They conclude that the evidence from these NIMH-funded studies is pharmacological treatments do not result in sustained benefit for the vast majority of ADHD children and thus do not warrant being the first option for treating ADHD.

In fact, what evidence is available during the followup phases found a deleterious effect from the ongoing use of stimulant medications to treat ADHD. Jensen et al. (2007) reported that in the 22-month MTA follow-up, "medication use was a significant marker, not of beneficial outcome, but of deterioration" (p. 996); and Molina et al. (2009) noted in the final follow-up assessment that stimulant medication use "was associated with worse hyperactivity-impulsivity and ODD symptoms and CIS impairment at 6 years" (p. 488). Similarly, Riddle et al. (2013) reported in the PATS follow-up study, "medication treatment in the original PATS predicted higher ADHD symptom severity between follow-up years 3 and $6 "$ (p. 10); raising again the issue identified in the MTA follow-up assessments of the increased risk of harm resulting from ongoing stimulant medication treatment.

Currie, Stabile, and Jones (2014) provide additional evidence of the increased risk of harm by the use of stimulant medications to treat ADHD. These three economists studied the medium- and long-term impact of adding prescription drug insurance coverage in Quebec. The summary of their findings is as follows:

We find that the introduction of the prescription drug insurance program increased the use of stimulants in Quebec relative to the rest of Canada. However, we find no evidence that the performance of children with ADHD improved. In fact, the increase in medication use among children with $A D H D$ is associated with increases in the probability of grade repetition, lower math scores, and a deterioration in relationships with parents. When we turn to an examination of long-term outcomes, we find that increases in medication use are associated with increases in the probability that a child has ever suffered from depression and decreases in the probability of post-secondary education among girls ( $p$. 59).

This repeated pattern of the loss of efficacy and increased risk of harm in studies assessing the impact from the ongoing use of stimulant medications likely accounts for much of the dramatic 
increase in the prescribing of antipsychotics to children (Pigott \& Cannon, 2014). Olfson, Blanco, Liu, Wang, and Correll (2012) report that between 1993-1998 and 2005-2009, the rate of antipsychotics prescribed to children increased by over $750 \%$. They found that disruptive behavior disorders (primarily ADHD) were the most common diagnoses in children that were prescribed an antipsychotic medication, accounting for $63 \%$ of such cases; and that in $54.1 \%$ of the outpatient visits, whenever an antipsychotic was prescribed, there was also an ADHD medication prescribed to the same child. In the PATS study, by the third year (age seven), an antipsychotic had been added to $8.3 \%$ of the preschoolers' medication regimen (and for $10.7 \%$, a norepinephrine reuptake inhibitor) and by age $10,12.9 \%$ were taking an antipsychotic (and for $8.6 \%$, a selective serotonin reuptake inhibitor was added); adding further evidence that stimulant medications act as gateway drugs to more psychiatric drugs, in the often fruitless pursuit of a chemical cure. Thus, as summarized in Pigott and Cannon (2014):

When the documented adverse effects of stimulants on ADHD children's growth, neural functioning, and cardiovascular system (Graham et al., 2011) are combined with their lack of long-term efficacy and gateway effect to other psychiatric drugs, stimulant medications must be displaced from their current status as the primary firstline treatment for ADHD (p.9).

Pigott and Cannon (2014) review the extensive evidence documenting NFB as the best available first-line treatment for ADHD. This treatment is based on Sterman and colleagues research finding that when hungry cats were fed contingent upon the increase in $12-16 \mathrm{~Hz}$ neuronal activity in the sensorimotor cortex (subsequently named the sensory motor rhythm [SMR]) the cats "became very alert" and displayed "an almost intense cessation of movement," behaviors which are key deficits in children with ADHD (Sterman \& Wyrwicka, 1967, p. 149). Building on Sterman's findings and using a rigorous double-blind within-subject reversal design, Lubar and Shouse demonstrated that, through realtime feedback of SMR paired with operant conditioning, ADHD children learned to self-regulate SMR with the resulting improvements or worsening of their ADHD symptoms based on whether they were reinforced to increase or decrease their SMR activity level (Lubar \& Shouse, 1976; Shouse \& Lubar, 1979).
NFB's evidence-base has now grown to over 70 published studies which find it effective in treating ADHD's core symptoms with the vast majority of these studies using standardized protocols targeting either SMR, the theta/beta ratio, or slow cortical potential training. Meta-analyses have found these standardized protocols to be efficacious and specific in treating ADHD's core symptoms (Arns, de Ridder, Strehl, Breteler, \& Coenen, 2009), with medium-tolarge effect sizes in randomized controlled trials when compared to semi-active (e.g., EMG biofeedback) and fully-active (e.g., computerized cognitive training) treatments (Arns, Heinrich, \& Strehl, 2014). Furthermore, unlike stimulant medication, reports of adverse effects from NFB are uncommon. More importantly, no other ADHD treatment has demonstrated credible evidence of sustained benefit following treatment termination, whereas this is the consistent finding of NFB studies that included follow-up assessments at 6 months (Gevensleben et al., 2010; Leins et al., 2007; Meisel, Servera, Garcia-Banda, Cardo, \& Moreno, 2013; Steiner, Frenette, Rene, Brennan, \& Perrin, 2014; Strehl et al., 2006) and 2 years (Gani, Birbaumer, \& Strehl, 2008; Monastra, 2005).

\section{Conclusions and Recommendations}

Psychopharmacology's increasingly acknowledged $50+$ year failure to identify any new molecular targets and to meaningfully improve outcomes, combined with the growing recognition that for many patients these medications cause more harm than benefit when used over time (e.g., Gøtzsche, 2015; Whitaker, 2010; Whitaker \& Cosgrove, 2015), provides an opportunity for neuroregulation treatments to gain widespread acceptance. Critical to gaining such acceptance is for the NFB and neuromodulation professional communities to maintain high scientific and clinical standards of practice. In recent years, high-quality NFB research has been published in mainstream peer-reviewed journals such as Pediatrics (e.g., Steiner et al., 2014; Strehl et al., 2006), the Journal of Attention Disorders (e.g., Mayer, Wyckoff \& Strehl, 2013; Wigton \& Krigbaum, 2015), and Biological Psychology with their entire January 2014 issue dedicated to NFB. While Insel argues that psychiatry needs to be remade into the discipline of clinical neuroscience (Insel, 2009; Insel \& Quirion, 2005), from inception that is our discipline. Thus, it is necessary to protect its validity and grow it through close adherence to high standards. 
Given psychopharmacology's crisis and the growing recognition of neuroregulation interventions' effectiveness and promise, there has been a significant increase in interest and investments in the field. This is a blessing and a curse. On the one hand, it funds increased research and development efforts to build more effective and user-friendly treatments, thereby accelerating acceptance. On the other hand, it increases outside scrutiny while simultaneously opening the gates for opportunists to make unsubstantiated claims for their products and methods and thereby tarnish the field. Therefore, it is important to establish a culture of responsibility, evidenced by a willingness to learn from failure and the courage to call out unsubstantiated claims. Professional NFB and neuromodulation membership societies need to foster such a culture or else we too may find ourselves in a crisis similar to that which psychopharmacology finds itself in today.

\section{Acknowledgements}

I would like to thank Dr. Genomary Krigbaum for her invaluable suggestions and edits that significantly improved the paper, although she may not agree with all of its content. In particular, I am grateful that her diligence kept me from making at least one if not more substantive errors.

\section{Statement of Competing Interests}

Dr. Pigott has consulted for Amen Clinics, Brain Resources, CNS Response, the International Society of Neurofeedback and Research, and Neuronetics. He also owns stock options in CNS Response, a company that markets a qEEG database to predict medication response.

\section{References}

Arns, M., de Ridder, S., Strehl, U., Breteler, M., \& Coenen, A. (2009). Efficacy of neurofeedback treatment in ADHD: The effects on inattention, impulsivity and hyperactivity: A metaanalysis. Clinical EEG and Neuroscience, 40(3), 180-189. http://dx.doi.org/10.1177/155005940904000311

Arns, M., Heinrich, H., \& Strehl, U. (2014). Evaluation of neurofeedback in ADHD: The long and winding road. Biological Psychology, 95, 108-115. http://dx.doi.org/10.1016 /j.biopsycho.2013.11.013

Bowden, C. L., Perlis, R. H., Thase, M. E., Ketter, T. A., Ostacher, M. M., Calabrese, J. R., ... Sachs, G. S. (2012). Aims and results of the NIMH systematic treatment enhancement program for bipolar disorder (STEP-BD). CNS Neuroscience \& Therapeutics, 18(3), 243-249. http://dx.doi.org/10.1111 j.1755-5949.2011.00257.x

Cantor, D. S., \& Stevens, E. (2009). QEEG correlates of auditoryvisual entrainment treatment efficacy of refractory depression. Journal of Neurotherapy, 13(2), 100-108. http://dx.doi.org $/ 10.1080 / 10874200902887130$

Choi, S. W., Chi, S. E., Chung, S. Y., Kim, J. W., Ahn, C. Y., \& $\mathrm{Kim}, \mathrm{H}$. T. (2011). Is alpha wave neurofeedback effective with randomized clinical trials in depression? A pilot study. Neuropsychobiology, 63(1), 43-51. http://dx.doi.org /10.1159/000322290

Currie, J., Stabile, M., \& Jones, L. (2014). Do stimulant medications improve educational and behavioral outcomes for children with ADHD? Journal of Health Economics, 37, 58-69. http://dx.doi.org/10.1016/j.jhealeco.2014.05.002

DePaulo, J. R. (2006). Bipolar disorder treatment: An evidencebased reality check. The American Journal of Psychiatry, 163(2), 175-176. http://dx.doi.org/10.1176/appi.ajp.163.2.175

Fava, M., Rush, A. J., Trivedi, M. H., Nierenberg, A. A., Thase, M. E., Sackeim, H. A., ... Kupfer, D. J. (2003). Background and rationale for the sequenced treatment alternatives to relieve depression (STAR* D) study. Psychiatric Clinics of North America, 26(2), 457-494.

Fibiger, H. C. (2012). Psychiatry, the pharmaceutical industry, and the road to better therapeutics. Schizophrenia Bulletin, 38(4), 649-650. http://dx.doi.org/10.1093/schbul/sbs073

Gani, C., Birbaumer, N., \& Strehl, U. (2008). Long term effects after feedback of slow cortical potentials and of theta-betaamplitudes in children with attention-deficit/hyperactivity disorder (ADHD). International Journal of Bioelectromagnetism, 10(4), 209-232.

Gevensleben, H., Holl, B., Albrecht, B., Schlamp, D., Kratz, O., Studer, P., ... Heinrich H. (2010). Neurofeedback training in children with ADHD: 6-Month follow-up of a randomized controlled trial. European Child \& Adolescent Psychiatry, 19(9), 715-724. http://dx.doi.org/10.1007/s00787-010-0109-5

Gøtzsche, P. C. (2015). Does long term use of psychiatric drugs cause more harm than good? Yes. British Medical Journal, 350, h2435. http://dx.doi.org/10.1136/bmj.h2435

Graham, J., Banaschewski, T., Buitelaar, J., Coghill, D., Danckaerts, M., Dittmann, R. W., ... Taylor, E. (2011). European guidelines on managing adverse effects of medication for ADHD. European Journal of Child \& Adolescent Psychiatry, 20(1), 17-37. http://dx.doi.org /10.1007/s00787-010-0140-6

Greden, J. F. (2013). Workplace depression: Personalize, partner, or pay the price. The American Journal of Psychiatry, 170(6), 578-581. http://dx.doi.org/10.1176/appi.ajp.2012.13030382

Greenberg, G. (2013, September 3). The psychiatric drug crisis. The New Yorker. Retrieved from http://www.newyorker.com /tech/elements/the-psychiatric-drug-crisis

Hickey, P. (2014, June 6). Psychiatry DID promote the chemical imbalance theory. Mad in America. Retrieved from http://www.madinamerica.com/2014/06/psychiatry-promotechemical-imbalance-theory/

Hyman, S. E. (2012). Revolution stalled. Science Translational Medicine, 4(155), $155 \mathrm{~cm} 11 . \quad \mathrm{http} / / / \mathrm{dx}$.doi.org/10.1126 /scitranslmed.3003142

Insel, T. R. (2009). Disruptive insights in psychiatry: Transforming a clinical discipline. The Journal of Clinical Investigation, 119(4), 700-705. http://dx.doi.org/10.1172/JCl38832

Insel, T. R., \& Quirion, R. (2005). Psychiatry as a clinical neuroscience discipline. Journal of the American Medical Association, 294(17), 2221-2224. http://dx.doi.org/10.1001 /jama.294.17.2221

Jensen, P. S., Arnold, L. E., Swanson, J. M., Vitiello, B., Abikoff, H. B., Greenhill, L. L., ... Hur, K. (2007). 3-year follow-up of the NIMH MTA study. Journal of the American Academy of Child \& Adolescent Psychiatry, 46(8), 989-1002. http://dx.doi.org/10.1097/CHI.0b013e3180686d48

John, E. R., Prichep, L. S., Friedman, J., \& Eastman, P. (1988). Neurometrics: Computer-assisted differential diagnosis of brain dysfunctions. Science, 293(4836), 162-169.

John, E. R., Prichep, L. S., Winterer, G., Herrmann, W. M., diMichele, F., Halper, J., ... Cancro, R. (2007) Electrophysiological subtypes of psychotic states. Acta Psychiatrica Scandinavica, 116(1), 17-35.

Kantor, E. D., Rehm, C. D., Haas, J. S., Chan, A. T., \& Giovannucci, E. L. (2015). Trends in prescription drug use 
among adults in the United States from 1999-2012. Journal of the American Medical Association, 314(17), 1818-1830. http://dx.doi.org/10.1001/jama.2015.13766

Kelland, K. (2017, January 16). No new antidepressants in sight despite growing need, experts warn. Reuters. Retrieved from www.reuters.com/article/us-health-antidepressantsidUSKBN14V2AQ

Leins, U., Goth, G., Hinterberger, T., Klinger, C., Rumpf, N., \& Strehl, U. (2007). Neurofeedback for children with ADHD: A comparison of SCP and Theta/Beta protocols. Applied Psychophysiology and Biofeedback, 32(2), 73-88. http://dx.doi.org/10.1007/s10484-007-9031-0

Lieberman, J. A., Stroup, T. S., McEvoy, J. P., Swartz, M. S., Rosenheck, R. A., Perkins, D. O., ... Hsiao, J. K. (2005). Clinical antipsychotic trials of intervention effectiveness (CATIE) investigators: Effectiveness of antipsychotic drugs in patients with chronic schizophrenia. The New England Journal of Medicine, 353(12), 1209-1223. http://dx.doi.org /10.1056/NEJMoa051688

Lubar, J. F., \& Shouse, M. N. (1976). EEG and behavioral changes in a hyperkinetic child concurrent with training of the sensorimotor rhythm (SMR): A preliminary report. Biofeedback and Self-Regulation, 1(3), 293-306. http://dx.doi.org /10.1007/bf01001170

Mayer, K., Wyckoff, S. N. \& Strehl, U. (2013). One size fits all? Slow cortical potentials neurofeedback. Journal of Attention Disorders, 17(5), 393-409. http://dx.doi.org/10.1177 $/ 1087054712468053$

Meisel, V., Servera, M., Garcia-Banda, G., Cardo, E., \& Moreno, I. (2013). Neurofeedback and standard pharmacological intervention in ADHD: A randomized controlled trial with sixmonth follow-up. Biological Psychology, 94(1), 12-21. http://dx.doi.org/10.1016/j.biopsycho.2013.04.015

Molina, B. S. G., Hinshaw, S. P., Swanson, J. M., Arnold, L. E., Vitiello, B., Jensen, P. S., ... The MTA Cooperative Group. (2009). The MTA at 8 years: Prospective follow-up of children treated for combined-type ADHD in a multisite study. Journal of the American Academy of Child \& Adolescent Psychiatry, 48(5), 484-500. $\quad$ http://dx.doi.org/10.1097 /CHI.0b013e31819c23d0

Monastra, V. J. (2005). Electroencephalographic biofeedback (neurotherapy) as a treatment for attention deficit hyperactivity disorder: Rationale and empirical foundation. Child and Adolescent Psychiatric Clinics of North America, 14, 55-82. http://dx.doi.org/10.1016/j.chc.2004.07.004

MTA Cooperative Group. (1999). A 14-month randomized clinical trial of treatment strategies for attention-deficit/hyperactivity disorder. Archives of General Psychiatry, 56(12), 1073-1086.

MTA Cooperative Group. (2004a). National Institute of Mental Health multimodal treatment study of ADHD follow-up: 24month outcomes of treatment strategies for attentiondeficit/hyperactivity disorder. Pediatrics, 113(4), 754-761.

MTA Cooperative Group. (2004b). National Institute of Mental Health multimodal treatment study of ADHD follow-up: Changes in effectiveness and growth after the end of treatment. Pediatrics, 113(4), 762-769.

Olfson, M., Blanco, C., Liu, S.-M., Wang, S., \& Correll, C. U. (2012). National trends in the office-based treatment of children, adolescents, and adults with antipsychotics. Archives of General Psychiatry, 69(12), 1247-1256. http://dx.doi.org/10.1001/archgenpsychiatry.2012.647

Peeters, F., Oehlen, M., Ronner, J., van Os, J., \& Lousberg, R. (2014). Neurofeedback as a treatment for major depressive disorder-A pilot study. PloS ONE, 9(3), e91837. http://dx.doi.org/10.1371/journal.pone.0091837

Pies, R. W. (2011, July 11). Psychiatry's new brain-mind and the legend of the "chemical imbalance." Psychiatric Times. Retrieved from http://www.psychiatrictimes.com/blogs/couchcrisis/psychiatry-new-brain-mind-and-legend-chemicalimbalance
Pigott, H. E. (2011). STAR*D: A tale and trial of bias. Ethical Human Psychology and Psychiatry, 13(1), 6-28. http://dx.doi.org/10.1891/1559-4343.13.1.6

Pigott, H. E. (2015). The STAR* D trial: It is time to reexamine the clinical beliefs that guide the treatment of major depression. Canadian Journal of Psychiatry [Revue Canadienne De Psychiatrie], 60(1), 9-13.

Pigott, H. E., \& Alter, G. S. (2011). In response to 'Do pharmacotherapy and/or psychotherapy work in depression? It depends!' and 'Widespread methodological problems limit validity of meta-analytic results'. Psychotherapy and Psychosomatics, 80(4), 247-248. http://dx.doi.org/10.1159 1000323614

Pigott, H. E., \& Cannon, R. (2014). Neurofeedback is the best available first-line treatment for ADHD: What is the evidence for this claim? NeuroRegulation, 1(1), 4-23. http://dx.doi.org /10.15540/nr.1.1.4

Pigott, H. E., Leventhal, A. M., Alter, G. S., \& Boren, J. J. (2010). Efficacy and effectiveness of antidepressants: Current status of research. Psychotherapy and Psychosomatics, 79(5), 267279. http://dx.doi.org/10.1159/000318293

Riddle, M. A., Yershova, K., Lazzaretto, D., Paykina, N., Yenokyan, G., Greenhill, L., ... Posner, K. (2013). The preschool attention-deficit/hyperactivity disorder treatment study (PATS) 6-year follow-up. Journal of the American Academy of Child \& Adolescent Psychiatry, 52(3), 264-278. http://dx.doi.org/10.1016/j.jaac.2012.12.007

Rush, A. J. (2002). Sequenced Treatment Alternatives to Relieve Depression $\left(S T A R^{*} D\right)$ research protocol. Received October 1, 2010, from the National Institute of Mental Health, following a request under the Freedom of Information Act. Mad in America. Retrieved from https://www.madinamerica.com/wp-content/uploads/2011/12 ISTAR-D\%20Protocol\%20with\%20Analytic\%20Plan.pdf

Rush, A. J., Trivedi, M. H., Wisniewski, S. R., Nierenberg, A. A., Stewart, J. W., Warden, D., ... Fava, M. (2006). Acute and longer-term outcomes in depressed outpatients requiring one or several treatment steps: A STAR* D report. American Journal of Psychiatry, 163(11), 1905-1917. http://dx.doi.org /10.1176/appi.ajp.163.11.1905

Shouse, M. N., \& Lubar, J. F. (1979). Operant conditioning of EEG rhythms and ritalin in the treatment of hyperkinesis. Biofeedback and Self-Regulation, 4(4), 299-312. http://dx.doi.org/10.1007/bf00998960

Steiner, N. J., Frenette, E. C., Rene, K. M., Brennan, R. T., \& Perrin, E. C. (2014). In-school neurofeedback training for ADHD: Sustained improvements from a randomized control trial. Pediatrics, 133(3), 483-492. http://dx.doi.org/10.1542 /peds.2013-2059

Sterman, M. B., \& Wyrwicka, W. (1967). EEG correlates of sleep: Evidence for separate forebrain substrates. Brain Research, $6(1), 143-163$.

Strehl, U., Leins, U., Goth, G., Klinger, C., Hinterberger, T., \& Birhaumer, N. (2006). Self-regulation of slow cortical potentials: A new treatment for children with attentiondeficit/hyperactivity disorder. Pediatrics, 118(5), 1530-1540. http://dx.doi.org/10.1542/peds.2005-2478

Trivedi, M. H., Rush, A. J., Wisniewski, S. R., Nierenberg, A. A., Warden, D., Ritz, L., ... STAR*D Study Team. (2006). Evaluation of outcomes with citalopram for depression using measurement-based care in STAR* D: Implications for clinical practice. The American Journal of Psychiatry, 163(1), 28-40. http://dx.doi.org/10.1176/appi.ajp.163.1.28

Trivedi, M. H., Stegman, D., Rush, A. J., Wisniewski, S. R., \& Nierenberg, A. A. (2002, July 31). STAR* $D$ clinical procedures manual. Retrieved from https://www.madinamerica.com/wp-content/uploads/2011/12 ISTAR_D Clinical Procedures Manual.htm 
Whitaker, R. (2010). Anatomy of an Epidemic: Magic Bullets, Psychiatric Drugs, and the Astonishing Rise of Mental IIIness in America. New York, NY: Crown Publishers.

Whitaker, R., \& Cosgrove, L. (2015). Psychiatry Under the Influence: Institutional Corruption, Social Injury, and Prescriptions for Reform. London, UK: Palgrave Macmillan.

Wigton, N. L., \& Krigbaum, G. (2015). Attention, executive function, behavior, and electrocortical function, significantly improved with 19-channel z-score neurofeedback in a clinical setting. Journal of Attention Disorders. Advance online publication. http://dx.doi.org/10.1177/1087054715577135

Received: January 18, 2017

Accepted: January 31, 2017

Published: March 15, 2017 